\title{
Enteric media: pre-poured or in-house?
}

\author{
C A Persaud, S J Eykyn
}

\begin{abstract}
Aims-(1) To evaluate the performance of in-house and pre-poured commercially available enteric agar by challenge with a large number of positive clinical specimens. (2) To set the standard (critical independent evaluation) which new products should reach. (3) To publish this information, so that others can make informed decisions about enteric media.

Methods-Thirteen media of anonymous source were challenged with "known" positive stool samples.

Results-In-house desoxycholate citrate agar performed best for overall pathogen isolation rates, for shigella isolation rates, and for most pathogens available on primary culture.

Conclusions-Desoxycholate citrate agar made by our own laboratory yielded the most pathogens and proved the most effective.

(f Clin Pathol 1995;48:568-570)
\end{abstract}

Keywords: Enteric media, commercial media, in-house media, assessment.

We recently evaluated xylose lysine desoxycholate (XLD), hektoen enteric (HE), and desoxycholate citrate (DCA) agars ${ }^{1}$ and subsequently reduced the number of primary enteric plates used without compromising the detection of enteric pathogens. As a result of this study and in the light of the expansion of commercially available pre-poured media, we compared the performance of such media and our own. Most laboratories feel that in-house media, which may or may not undergo quality control evaluation, are better than commercially available media because they are fresher. Manufacturers apply strict quality control procedures and have thoroughly researched factors affecting storage and shelf life. What is lacking is critical independent evaluation of media when tested with clinical specimens rather than laboratory strains of bacteria.

\section{Methods}

One hundred and twenty known positive stool samples (excluding those known to be growing Campylobacter sp) received from NHS and Public Health Laboratory Service (PHLS) laboratories throughout the United Kingdom including St Thomas' Hospital were tested on 13 different media. Sources of media, including our in-house DCA, were made anonymous on receipt by administration staff. All prepoured plates were stored at $4^{\circ} \mathrm{C}$, and packs remained unopened until the day of use. In-house DCA was made according to the manufacturer's instructions and the plates stored in the dark at room temperature and used within two weeks. Positive stool samples were received by post (in accordance with Post Office regulations) or courier and stored at $4^{\circ} \mathrm{C}$. The age of samples varied from 48 hours to four weeks, with the majority at least one week old. In order to manage the workload most effectively, 20 samples were inoculated on all test plates available on the Tuesday of each week. Telephone enquiries were made if plates had not arrived by this time and reasons for delay noted.

Technical work was performed by two state registered medical laboratory scientific officers (MLSO), who were given details of the appearance of non-lactose fermenters on the different types of media used. Specimens were inoculated on to 13 media (A-M) and enriched in selenite broth (Unipath BO164A-all of the same batch) which was subcultured on to the 13 test plates. Plates were inoculated consecutively with a loaded cotton wool swab to make a pool. The swab was reloaded after every second plate to reduce inoculum bias. Selenite broth was inoculated with a pea sized amount of stool specimen (or $1 \mathrm{ml}$ if liquid). Plates and selenite broth were incubated overnight at $37^{\circ} \mathrm{C}$. A standard loopful $(5 \mu \mathrm{l})$ of enrichment broth was inoculated to form a pool on each plate and streaked out for single colonies. Plates were incubated overnight at $37^{\circ} \mathrm{C}$. Non-lactose fermenters were identified with API 10S (BioMerieux) and confirmed serologically where necessary. Shigellas were additionally confirmed with API 20E (Bio-Merieux). Where a pathogen with the same API profile was isolated from multiple plates, serological confirmation was only performed on one random positive plate. Salmonellas that agglutinated with 0:9 and H:g antisera were called Salmonella enter-

Table 1 Pathogens isolated from 90 positive stool specimens

\begin{tabular}{lr}
\hline S enteritidis & 27 \\
S typhimurium & 10 \\
S virchow & 5 \\
S hadar & 3 \\
S heidelberg & 3 \\
S bredeney & 2 \\
S brandenberg & 1 \\
S colindale & 1 \\
S durban & 1 \\
S emek & 1 \\
S gold coast & 1 \\
Sh sonnei & 29 \\
Sh dysenteriae & 2 \\
Sh flexneri & 2 \\
Sh boydii & 1 \\
Plesiomonas shigelloides & 2 \\
Yersinia enterocolitica & 1 \\
Total & 92 \\
\hline
\end{tabular}

Two specimens contained a mixture of pathogens, one $S$ enteritidis and $S h$ dysenteriae, the other $S$ enteritidis and Plesiomonas shigelloides.
Department, St Thomas' Hospital, C A Persaud 
Table 2 Comparison of overall pathogen isolation rates from 13 media

\begin{tabular}{|c|c|c|c|c|c|}
\hline Plate & Medium & No. of batches & Total pathogens & Salmonella & Shigella \\
\hline $\begin{array}{l}\text { A } \\
\text { B } \\
\text { C } \\
\text { D } \\
\text { E } \\
\text { F } \\
\text { G } \\
\text { H } \\
\text { J } \\
\text { K } \\
\text { M } \\
\text { N } \\
\text { O }\end{array}$ & $\begin{array}{l}\text { DCA } \\
\text { DCA } \\
\text { DCA } \\
\text { DCA } \\
\text { DCA } \\
\text { DCAH } \\
\text { DCLS } \\
\text { DCLS } \\
\text { DCLS } \\
\text { HE } \\
\text { HE } \\
\text { XID } \\
\text { XLD }\end{array}$ & $\begin{array}{l}4 \\
5 \\
3 \\
7 \\
3 \\
5 \\
2 \\
3 \\
3 \\
4 \\
3 \\
6 \\
6\end{array}$ & $\begin{array}{r}89 \%(55 / 62) \\
100 \%(92 / 92) \\
89 \%(82 / 92) \\
85 \%(67 / 79) \\
97 \%(89 / 92) \\
98 \%(90 / 92) \\
87 \%(68 / 78) \\
96 \%(88 / 92) \\
93 \%(86 / 92) \\
92 \%(85 / 92) \\
95 \%(87 / 92) \\
93 \%(86 / 92) \\
92 \%(85 / 92)\end{array}$ & $\begin{array}{r}98 \%(40 / 41) \\
100 \%(55 / 55) \\
98 \%(54 / 55) \\
93 \%(43 / 45) \\
100 \%(55 / 55) \\
100 \%(55 / 55) \\
98 \%(45 / 46) \\
100 \%(55 / 55) \\
98 \%(54 / 55) \\
100 \%(55 / 55) \\
100 \%(55 / 55) \\
98 \%(54 / 55) \\
100 \%(55 / 55)\end{array}$ & $\begin{array}{r}68 \%(13 / 19) \\
100 \%(34 / 34) \\
76 \%(26 / 34) \\
71 \%(22 / 31) \\
94 \%(32 / 34) \\
97 \%(33 / 34) \\
77 \%(23 / 30) \\
94 \%(32 / 34) \\
91 \%(31 / 34) \\
85 \%(29 / 34) \\
88 \%(30 / 34) \\
91 \%(31 / 34) \\
88 \%(30 / 34)\end{array}$ \\
\hline
\end{tabular}

DCA = desoxycholate citrate agar; DCAH = desoxycholate citrate agar (Hynes modification); DCLS = desoxycholate citrate lactose sucrose agar; $\mathrm{HE}=$ hektoen enteric agar; $\mathrm{XLD}=$ xylose lysine desoxycholate agar.

itidis and those with $0: 4 \mathrm{H}: \mathrm{i}, S$ typhimurium. Other salmonellas were sent to the Laboratory of Enteric Pathogens (CPHL, Colindale) for identification. All pathogens were saved in glycerol broth at $-70^{\circ} \mathrm{C}$

\section{Results}

Of 120 stool specimens, $102(85 \%)$ were received from other laboratories and the remainder were from St Thomas' Hospital. Not all samples yielded pathogens (90/120), and two samples yielded two pathogens. Table 1 shows the pathogens isolated. Not all media were available throughout the six week period: agar types A, D and $G$ were available for 4,5 , and 5 weeks respectively, and therefore the performance of each has been given as a percentage of the pathogens isolated during that time. For this reason the $p$ value for the null hypothesis could not be applied. Statistical analysis using $95 \%$ binomial probability confidence levels showed strong evidence of

Table 3 Comparison of isolation rates from primary culture plates

\begin{tabular}{lllll}
\hline Plate & Medium & Total pathogens & Salmonella & Shigella \\
\hline A & DCA & $45 \%(28 / 62)$ & $37 \%(15 / 41)$ & $58 \%(11 / 19)$ \\
B & DCA & $84 \%(77 / 92)$ & $80 \%(44 / 55)$ & $88 \%(30 / 34)$ \\
C & DCA & $58 \%(53 / 92)$ & $64 \%(35 / 55)$ & $50 \%(17 / 34)$ \\
D & DCA & $49 \%(39 / 79)$ & $51 \%(23 / 45)$ & $48 \%(15 / 31)$ \\
E & DCA & $74 \%(68 / 92)$ & $73 \%(40 / 55)$ & $76 \%(26 / 34)$ \\
F & DCAH & $74 \%(68 / 92)$ & $67 \%(37 / 55)$ & $88 \%(30 / 34)$ \\
G & DCLS & $54 \%(42 / 78)$ & $67 \%(31 / 46)$ & $57 \%(17 / 30)$ \\
H & DCLS & $82 \%(75 / 92)$ & $84 \%(46 / 55)$ & $85 \%(29 / 34)$ \\
J & DCLS & $61 \%(56 / 92)$ & $60 \%(33 / 55)$ & $65 \%(22 / 34)$ \\
K & HE & $63 \%(58 / 92)$ & $60 \%(33 / 55)$ & $71 \%(24 / 34)$ \\
M & HE & $68 \%(63 / 92)$ & $69 \%(38 / 55)$ & $71 \%(24 / 34)$ \\
N & XLD & $68 \%(63 / 92)$ & $67 \%(37 / 55)$ & $74 \%(25 / 34)$ \\
O & XLD & $65 \%(60 / 92)$ & $71 \%(39 / 55)$ & $68 \%(23 / 34)$ \\
\hline
\end{tabular}

$\mathrm{DCA}=$ desoxycholate citrate agar; $\mathrm{DCAH}=$ desoxycholate citrate agar (Hynes modification) $\mathrm{DCLS}=$ desoxycholate citrate lactose sucrose agar; $\mathrm{HE}=$ hektoen enteric agar; $\mathrm{XLD}=\mathrm{xylose}$ lysine desoxycholate agar.

Table 4 Comparison of isolation rates post-selenite enrichment culture

\begin{tabular}{lllrl}
\hline Plate & Medium & Total pathogens & Salmonella & \multicolumn{1}{l}{ Shigella } \\
\hline A & DCA & $77 \%(48 / 62)$ & $98 \%(40 / 41)$ & $42 \%(8 / 19)$ \\
B & DCA & $89 \%(82 / 92)$ & $100 \%(55 / 55)$ & $79 \%(27 / 34)$ \\
C & DCA & $79 \%(73 / 92)$ & $96 \%(53 / 55)$ & $59 \%(20 / 34)$ \\
D & DCA & $75 \%(59 / 79)$ & $96 \%(43 / 45)$ & $55 \%(17 / 31)$ \\
E & DCA & $87 \%(80 / 92)$ & $100 \%(55 / 55)$ & $74 \%(25 / 34)$ \\
F & DCAH & $84 \%(77 / 92)$ & $100 \%(55 / 55)$ & $71 \%(24 / 34)$ \\
G & DCLS & $78 \%(61 / 78)$ & $98 \%(45 / 46)$ & $63 \%(19 / 30)$ \\
H & DCLS & $85 \%(78 / 92)$ & $100 \%(55 / 55)$ & $74 \%(25 / 34)$ \\
J & DCLS & $82 \%(75 / 92)$ & $98 \%(54 / 55)$ & $74 \%(25 / 34)$ \\
K & HE & $82 \%(75 / 92)$ & $100 \%(55 / 55)$ & $68 \%(23 / 34)$ \\
M & HE & $80 \%(74 / 92)$ & $100 \%(55 / 55)$ & $65 \%(22 / 34)$ \\
N & XLD & $83 \%(76 / 92)$ & $98 \%(54 / 55)$ & $71 \%(24 / 34)$ \\
O & XLD & $83 \%(76 / 92)$ & $100 \%(55 / 55)$ & $71 \%(24 / 34)$ \\
\hline
\end{tabular}

DCA $=$ desoxycholate citrate agar; DCAH $=$ desoxycholate citrate agar (Hynes modification) DCLS = desoxycholate citrate lactose sucrose agar; $\mathrm{HE}=$ hektoen enteric agar; $\mathrm{XLD}=\mathrm{xylos}$ lysine desoxycholate agar. differences in performance in overall pathogen isolation rates, as well as the isolation rate of shigellas and other pathogens available on primary culture.

Over the six week period, between two and seven different batches of each product were tested. Table 2 shows the overall performance for each medium for enteric pathogens, as a percentage of isolates; table 3 shows the isolation rates on primary media only; and table 4 shows the isolation rates after selenite subculture.

\section{Discussion}

This controlled study assessed the performance of in-house and commercially available prepoured enteric media when challenged with clinical specimens. Stool specimens known to have yielded a pathogen were requested from other laboratories to ensure sufficient numbers of stools necessary for this study and remove the bias of pathogens known to grow on our medium. Failure to isolate a pathogen from every specimen was attributed to the effect of transit, or storage at $4^{\circ} \mathrm{C}$. Morris et al compared shigella isolation on direct culture after storage in transport media (varying the length of time held in transport medium) and found that recovery of shigellas fell quickly regardless of transport medium used. No attempt was made to salvage pathogens and it was accepted that some specimens would be negative. Specimens were processed blindly and not correlated with original findings.

We were surprised that despite advanced notice of this project with a stipulated requirement of 40 test plates per week for six weeks, three manufacturers were unable to supply plates. Reasons given included failure of quality control and administration problems. Although we did not test specifically for interbatch variation, this must be reflected in our results. Since all manufacturers issued plates after successful quality control tests we have assumed that interbatch variation was within acceptable limits.

The overall pathogen isolation rates for the different media varied between $85 \%$ and $100 \%$. Even greater disparity was shown in the isolation rate of shigellas (68-100\%). Media traditionally included for shigellas such as hektoen and XLD performed unfavourably compared to one DCA plate (B). The performance of DCA based media varied widely (depending 
on source), particularly with shigellas. Failure to isolate shigellas was probably due to overgrowth of other flora, but it is difficult to produce a medium which inhibits normal flora, yet allows shigellas to flourish. In our previous study ${ }^{1}$ we established that $90 \%$ of laboratories used the same medium for primary and secondary culture and thus the results shown in table 2 are applicable to most laboratories. Obviously DCA based media are not all the same and we should seek critical evaluation of media before changing. The two types of $\mathrm{XLD}$ gave similar results, as did the two types of hektoen. There was wide disparity in the isolation rates on primary media (45-84\%), yet early detection and identification of pathogens of clinical importance was essential, both for treatment and for infection control. Salmonellas presented little challenge to the media after enrichment in selenite broth.

Many manufacturers quote a shelf life of four to six weeks for their pre-poured plates. Our in-house plate has a shelf life of two weeks and is stored at room temperature in the dark to prevent the development of the red precipitate (sodium desoxycholate). Plates B and E were made from different batches of the same powder, one made by the manufacturer $(E)$, the other being our in-house DCA (B). Both performed well despite differences in storage conditions, shelf life, and quality control tests. Ironically, we perform only minimal quality control (sterility test) on our in-house media and yet achieved a satisfactory performance. Manufacturers often perform stringent quality control checks, usually with pure cultures. Quality control should ideally be performed with clinical specimens, since ability to grow an organism in pure culture may not predict performance with clinical samples. Since plates $B$ and $E$ are essentially the same DCA, the difference in isolation rates $(3 \%)$ must be attributed to different factors such as storage conditions, shelf life, reproducibility of inoculum, and interbatch variation. If we accept that such factors do have an effect, we would have expected all other media to achieve $97 \%$ success rates; wider differences cannot be explained.

The limitation of this study is that our inhouse medium (B) gave these results in our hands, and the quality of in-house plates will vary depending on personnel, storage, and shelf life assigned. However, the performance of STH in-house and pre-poured media DCA (B and $E$ respectively) correlated well.

This evaluation has taken considerable resources: two full time state registered MLSOs for six weeks and $£ 2200$ in consumables. Such resources are rarely available in diagnostic laboratories, yet this is the only way to assess the performance of different media!

Manufacturers are often keen to promote enteric agars that are specific for salmonellas and contain biochemicals which produce characteristic colonies, such as Rambach agar. ${ }^{23}$ This innovative new agar is so specific for salmonellas that it could only ever be a second primary plate in diagnostic microbiology. Rambach agar costs approximately 10 times as much as standard enteric plates, and may be useful to the food industry, but in diagnostic microbiology laboratories, where a variety of pathogens is encountered, resources may be better spent on critical evaluation of media commonly in use.

This study supports the need for legislation to standardise quality control tests. Enteric media should be challenged with clinical samples to test their efficacy before the product is released. This is already done by at least one company who supplied us with media.

Over this six week project despite advanced warning several companies were unable to provide media on time, a cautionary note for laboratories considering purchasing pre-poured media.

Our previous study ${ }^{1}$ showed that most laboratories surveyed used two or more primary plates for routine culture. We isolated all pathogens from a single plate (B), supporting our choice of medium for the isolation of enteric pathogens. Changes of media are frequently the result of failure to isolate an NEQAS pathogen, and an extra plate is added to "be on the safe side" (overcompensation for an artificial test?) or because we "feel" that we should not rely on one enteric plate, and a member of staff has "experience" of an alternative medium. Alternatively a new or cheaper product may be launched and we are so impressed with the product that we perhaps run it in parallel for a week or two and then incorporate it into the routine. Surely as consumers we should demand critical independent assessment. All can benefit from such information.

We thank the following: bio-Merieux, Becton-Dickinson, Cherwell Laboratories, Difco, Merck, Unipath, and our own media well faboratories, Difco, Merck, Unipath, and our own media Miss Minal Bhanot BSc for the technical work; Miss Kay Roast Miss Minal Bhanot BSc for the technical work; Miss Kay Roast
for administration assistance; Ashford PHL, Brighton PHL, for administration assistance; Ashford PHL, Brighton PHL,
Bristol PHL, Cambridge PHL, Chelsea and Westminster HosBristol PHL, Cambridge PHL, Chelsea and Westminster Hospital, Guy's Hospital, Kent and Canterbury Hospital, Luton and Dunstable PHL, Manchester PHL, Newcastle PHL, Nottingham PHL, Oxford PHL, Plymouth PHL, Poole PHL and Tooting PHL, for providing positive stool specimens; CPHL Laboratory of Enteric Pathogens, Colindale, for serotyping Phillips, without whose support this project would not have been possible.

1 Persaud C, Eykyn S J. Stool culture: value for money? $\mathcal{F}$ Clin Pathol 1994;47:790-2.

2 Grunewald R, Henderson R, Yappow S. Use of Rambach propylene glycol containing agar for identification of Salmonella spp. F Clin Microbiol 1991;29:2354-6.

3 Abdalla S, Vila J, Jimenez de Anta M T. Identification of Salmonella spp. with Rambach agar in conjunction with the 4-methylumbelliferyl caprylate (MUCAP) fluorescence test. Br $\mathcal{Y}$ Biomed Sci 1994;51:5-8.

4 Morris G K, Koehler J A, Gangarosa E J, Sharrar R G. Comparison of media for direct isolation and transport of shigellae from faecal specimens. Appl Microbiol 1970;19: 434-7. 